\title{
Inadequate Brain Glycogen or Sleep Increases Spreading Depression Susceptibility
}

\author{
Kivilcim Kilic, MD, PhD, ${ }^{1 \star ~ H u l y a ~ K a r a t a s, ~ M D, ~ P h D, ~}{ }^{1 \star}$ Buket Dönmez-Demir, PhD, ${ }^{1}$ \\ Emine Eren-Kocak, MD, PhD, ${ }^{1}$ Yasemin Gursoy-Ozdemir, MD, PhD, ${ }^{1,7}$ \\ Alp Can, MD, PhD, ${ }^{2}$ Jean-Marie Petit, PhD, ${ }^{3}$ \\ Pierre J. Magistretti, MD, $\mathrm{PhD}^{4,5}$ and Turgay Dalkara, MD, $\mathrm{PhD}^{6}$
}

\begin{abstract}
Objective: Glycogen in astrocyte processes contributes to maintenance of low extracellular glutamate and $\mathrm{K}^{+}$concentrations around excitatory synapses. Sleep deprivation (SD), a common migraine trigger, induces transcriptional changes in astrocytes, reducing glycogen breakdown. We hypothesize that when glycogen utilization cannot match synaptic energy demand, extracellular $\mathrm{K}^{+}$can rise to levels that activate neuronal pannexin- 1 channels and downstream inflammatory pathway, which might be one of the mechanisms initiating migraine headaches.

Methods: We suppressed glycogen breakdown by inhibiting glycogen phosphorylation with 1,4-dideoxy-1,4-iminoD-arabinitol (DAB) and by SD.

Results: DAB caused neuronal pannexin-1 large pore opening and activation of the downstream inflammatory pathway as shown by procaspase-1 cleavage and HMGB1 release from neurons. Six-hour SD induced pannexin-1 mRNA. $\mathrm{DAB}$ and SD also lowered the cortical spreading depression (CSD) induction threshold, which was reversed by glucose or lactate supplement, suggesting that glycogen-derived energy substrates are needed to prevent CSD generation. Supporting this, knocking down the neuronal lactate transporter MCT2 with an antisense oligonucleotide or inhibiting glucose transport from vessels to astrocytes with intracerebroventricularly delivered phloretin reduced the CSD threshold. In vivo recordings with a $\mathrm{K}^{+}$-sensitive/selective fluoroprobe, Asante Potassium Green-4, revealed that $\mathrm{DAB}$ treatment or $\mathrm{SD}$ caused a significant rise in extracellular $\mathrm{K}^{+}$during whisker stimulation, illustrating the critical role of glycogen in extracellular $\mathrm{K}^{+}$clearance.

Interpretation: Synaptic metabolic stress caused by insufficient glycogen-derived energy substrate supply can activate neuronal pannexin-1 channels as well as lower the CSD threshold. Therefore, conditions that limit energy supply to synapses (eg, SD) may predispose to migraine attacks, as suggested by genetic studies associating glucose or lactate transporter deficiency with migraine.
\end{abstract}

ANN NEUROL 2018;83:61-73

G lycogen metabolism is tightly regulated in correlation with synaptic activity by a restricted number of neurotransmitters, including noradrenaline, vasoactive intestinal peptide, and adenosine. ${ }^{1-4}$ Pointing to its imperative role in synaptic function, glycogen has been shown to be crucial in the hippocampus for long-term memory formation. ${ }^{4}$ Glycogen is also needed for maintenance of low extracellular glutamate and $\mathrm{K}^{+}$concentrations around synapses especially during intense glutamatergic transmission. $^{5-7}$ The coupling of glycogen utilization with synaptic

View this article online at wileyonlinelibrary.com. DOI: 10.1002/ana.25122

Received Aug 20, 2016, and in revised form Nov 23, 2017. Accepted for publication Dec 12, 2017.

Address correspondence to Dr Dalkara, Department of Neurology, Faculty of Medicine, Institute of Neurological Sciences and Psychiatry, Hacettepe University, Sihhiye, Ankara, Turkey. E-mail address: tdalkara@hacettepe.edu.tr

*These authors contributed equally to this study.

From the ${ }^{1}$ Institute of Neurological Sciences and Psychiatry, Hacettepe University, Ankara, Turkey; ${ }^{2}$ Department of Histology and Embryology, School of Medicine, Ankara University, Ankara, Turkey; ${ }^{3}$ Center for Psychiatric Neuroscience, Department of Psychiatry, University Hospital of the Canton of Vaud (CHUV), Prilly, Switzerland; ${ }^{4}$ King Abdullah University of Science and Technology, Thuwal, Saudi Arabia; ${ }^{5}$ Brain Mind Institute, Federal Institute of Technology Lausanne (EPFL), Lausanne, Switzerland; ${ }^{6}$ Department of Neurology, Faculty of Medicine and Institute of Neurological Sciences and Psychiatry, Hacettepe University, Ankara, Turkey; and ${ }^{7}$ Current address for Dr Gursoy-Ozdemir: Department of Neurology, School of Medicine, Koç University, Istanbul, Turkey 
transmission appears so fundamental that even under conditions where the energy production via glucose breakdown is sufficient, glycogen is still required for glutamate uptake. $^{8}$

Increased extracellular $\mathrm{K}^{+}$, glutamate, and adenosine triphosphate (ATP) concentrations and intracellular $\mathrm{Ca}^{2+}$ rises can activate neuronal pannexin-1 (Panx-1) channels. ${ }^{9-13}$ The above conditions are present during cortical spreading depression (CSD), the putative cause of migraine aura. ${ }^{14,15}$ CSD has been shown to activate neuronal Panx-1 channels in the mouse brain in vivo, and this has been proposed as a potential mechanism initiating a parenchymal-meningeal inflammatory cascade that leads to activation of meningeal trigeminal afferents, and hence, headache following migraine aura. ${ }^{16,17}$ As similar conditions may emerge around intensely active excitatory synapses when glycogen-derived glucose and lactate cannot match the increased energy demand for restoration of the transmembrane gradients of glutamate, $\mathrm{K}^{+}$, and $\mathrm{Ca}^{2+}$, we hypothesize that neuronal Panx-1 channels can be activated, and this might be one of the potential mechanisms by which sleep deprivation, a well-known migraine trigger, ${ }^{18}$ can initiate the inflammatory cascade leading to headache without requiring aura (ie, without CSD).

Sleep deprivation (ie, extended wakefulness) induces astrocytic expression of the enzyme PTG (protein targeting to glycogen), a noncatalytic subunit of the protein phosphatase-1 that results in an increased activity of glycogen synthase, the enzyme responsible for incorporation of glucose into glycogen. ${ }^{19-21}$ Therefore, sleep deprivation may limit the capacity to maintain low extracellular glutamate and $\mathrm{K}^{+}$concentrations during sustained excitatory transmission by driving glycogen metabolism toward synthesis rather than its utilization as a glucose and lactate source. Hence, a decreased capacity for such compensation (eg, due to genetic predisposition or hormonal factors) may make some migraineurs more vulnerable to changes in sleep rhythm either by predisposing to synaptic conditions that can activate large pore channels such as neuronal Panx-1/P2X7 complex and the downstream inflammatory cascade (migraine without aura) or by lowering the threshold for CSD generation (migraine with aura). ${ }^{16,17}$ In both cases, Panx-1 channels may serve as sensors that detect the neuronal stress induced by metabolically uncoupled synaptic transmission or CSD and initiate the inflammatory cascade that can potentially activate the trigeminal system.

To test these hypotheses, we have investigated whether the impairment of glycogen-derived energy supply to synapses can directly activate Panx-1 channels or lower the CSD induction threshold in the mouse cortex in vivo. To reduce glycogen mobilization, we used a pharmacological inhibitor of glycogen phosphorylase, 1,4-dideoxy-1,4-imino-D-arabinitol (DAB). Because the energy metabolite transferred from astrocytes to neurons upon glycogen breakdown is lactate, ${ }^{22}$ we also used an antisense oligodeoxynucleotide (ODN) for the neuronal monocarboxylate transporter MCT2. ${ }^{4}$ Finally, we induced sleep deprivation of 6 hours to reduce glycogen availability for supporting excitatory synaptic transmission. We have found that these approaches reducing glycogen mobilization can initiate an inflammatory cascade downstream to Panx-1 complex by directly triggering caspase-1 cleavage and HMGB1 release from neurons even in the absence of CSD. ${ }^{16}$ We have also found that insufficient glycogen-derived synaptic energy supply can reduce the CSD threshold.

\section{Materials and Methods}

\section{Animals, Surgery, and Intracerebroventricular Injections}

Swiss albino mice $(n=209)$ weighing 25 to $36 \mathrm{~g}$ were housed under diurnal lighting conditions (12 hours darkness and 12 hours light) and fed ad libitum before the experiment. Animal housing, care, and application of experimental procedures were all carried out in accordance with the institutional guidelines and approved by the Hacettepe University Animal Experiments Local Ethics Committee (2009/38-2). Animals were anesthetized with urethane/xylazine $(1.25 \mathrm{~g} / \mathrm{kg}$ and $10 \mathrm{mg} / \mathrm{kg}$, intraperitoneal) during the experiments. Brief isoflurane (1-2\%) anesthesia was used for intracerebroventricular (i.c.v.) cannula placement a day before the experiment. Oxygen (2l/min) was supplied throughout the procedure. Body temperature was kept at $37.0 \pm 0.1^{\circ} \mathrm{C}$ with a homeothermic blanket and a rectal probe. Tissue oxygen saturation and pulse rate were monitored with a pulse oximeter. Mice were placed in a stereotactic frame after the induction of deep anesthesia. A frontal burr hole was drilled over the right hemisphere for CSD induction with an epidural KCl-soaked cotton ball.

For i.c.v. injections of propidium iodide (PI), DAB, and carbenoxolone (CBX), a Hamilton syringe with a 26-gauge needle was slowly inserted through the cannula placed a day before the experiment, except for the CSD-induction threshold experiments. For the threshold experiments, DAB or phloretin was slowly injected into the left ventricle with help of a micromanipulator within 8 to 10 minutes at the coordinates $0.1 \mathrm{~mm}$ posterior, $0.9 \mathrm{~mm}$ lateral, $3.1 \mathrm{~mm}$ deep with reference to bregma. DAB $(0.25 \mathrm{M}, 0.75 \mu \mathrm{l}$, dissolved in saline; SigmaAldrich, St Louis, MO) or saline $(0.75 \mu \mathrm{l})$ was injected $1 \mathrm{~h}$ before the threshold test. During the $1-\mathrm{h}$ waiting period, direct current (DC) potential changes were continuously monitored. Phloretin (2M, $1 \mu \mathrm{l}$; dissolved in dimethylsulfoxide [DMSO]; Sigma-Aldrich) or DMSO ( $1 \mu \mathrm{l})$ was injected 30 minutes before threshold experiments. All solutions were heated to $37^{\circ} \mathrm{C}$ before injection. 


\section{PI Injection}

We assessed the opening of Panx-1 channels with PI after DAB administration. PI is a membrane-impermeable dye; however, it can pass through large pore channels when they are open and give fluorescence by interacting with nucleic acids after entering the cell. A $1 \mathrm{~mm}$-thick (outer diameter) polyethylene cannula was slowly inserted $2.1 \mathrm{~mm}$ deep into the left lateral ventricle through a burr hole drilled over the left parietal bone under isoflurane anesthesia. The day after cannula implantation, animals were placed in a stereotaxic frame under urethane/xylazine anesthesia. PI $(0.5 \mu \mathrm{l}, 1 \mathrm{mg} / \mathrm{ml}$ in distilled water) was injected with a 26-gauge needle on a Hamilton syringe slowly inserted through the cannula by a micromanipulator 55 minutes after $\mathrm{DAB}$ or saline (as control for DAB experiments) injection. The tip of the needle was adjusted to target the ventricle $3.1 \mathrm{~mm}$ deep with reference to dura. Mice were sacrificed by transcardial perfusion with heparinized saline and $4 \%$ paraformaldehyde (PFA) 1 hour after DAB/saline injection $(n=3$ in each group). Brains were quickly removed and kept in $4 \%$ PFA overnight followed by $30 \%$ sucrose for 2 days. Twenty-micrometerthick sections were cut on a freezing cryostat and mounted in glycerol/phosphate-buffered saline (PBS; 1:1) medium containing $12.5 \mathrm{mg} / \mathrm{ml}$ sodium azide and $1 \mu \mathrm{l} / \mathrm{ml}$ Hoechst-33258. PI fluorescence was evaluated on 3 consecutive images of coronal brain sections under $\times 400$ magnification with appropriate filter sets using a fluorescence microscope (E600; Nikon, Tokyo, Japan).

\section{CBX Injection}

To inhibit Panx-1 channels, 400ng CBX (in $2 \mu$ distilled water, $\mathrm{n}=3$ ) was slowly injected into the left lateral ventricle with the needle of a Hamilton syringe driven by a micromanipulator through a polyethylene cannula placed a day before as described above. An equal volume of distilled water $(n=3)$ was injected to control animals. After 10 minutes, PI was slowly injected through the same cannula as described above to monitor Panx1 activation in both groups. Mice were sacrificed by transcardial perfusion with heparinized saline and 4\% PFA 5 minutes after PI injection, and the above tissue-processing protocol was applied for cryoprotection, sectioning, and evaluation of the PIpositive cells.

\section{Immunohistochemistry}

Because caspase- 1 is reportedly cleaved 5 minutes after Panx-1 activation, ${ }^{16}$ mice were sacrificed 65 minutes after $\mathrm{DAB} /$ saline injection ( $\mathrm{n}=3 /$ group). After cryoprotection, $40-\mu \mathrm{m}$-thick coronal sections were cut on a sliding microtome. As HMGB1 is reportedly released 30 minutes after Panx-1 activation, another set of mice was sacrificed 90 minutes after DAB injection. These mice were transcardially perfused with heparinized saline and $4 \%$ PFA. Brains were quickly removed, postfixed in the same solution overnight, and cryoprotected in 30\% sucrose solution for 2 days. Twenty-micrometer-thick coronal sections were cut on a freezing cryostat. Sections were immunostained with either goat polyclonal cleaved caspase-1 (1:200; Santa Cruz Biotechnology, Santa Cruz, CA), rabbit polyclonal
HMGB1 (1:200; Abcam, Cambridge, MA), or mouse monoclonal NeuN antibody (1:200; Chemicon International, Temecula, CA) followed by secondary labeling with donkey anti-goat IgG-FITC (1:200; Santa Cruz Biotechnology), goat anti-rabbit Cy2 antibody (1:200; Jackson ImmunoResearch, West Grove, PA), or goat anti-mouse Cy3 antibody (1:200, Jackson ImmunoResearch). Primary antibody omissions were performed to test the specificity of immunoreactivity. Sections were mounted in glycerol $/ \mathrm{PBS}(1: 1)$ medium containing $12.5 \mathrm{mg} / \mathrm{ml}$ sodium azide and $1 \mu \mathrm{l} / \mathrm{ml}$ Hoechst-33258, and were examined under a epifluorescence and laser-scanning confocal microscopes with appropriate filter sets.

\section{Cerebrospinal Fluid Collection and HMGB1 Western Blotting}

For the detection of HMGB1 release, cerebrospinal fluid (CSF) was collected through an i.c.v. cannula placed into left lateral ventricle a day before the experiment. One hour after DAB injection through the same cannula, 2.5 $\mu \mathrm{l}$ CSF was collected within 30 minutes ( $\mathrm{n}=3$ mice). For sham control experiments $(\mathrm{n}=3$ mice), saline was injected instead of DAB. A CSF sample was discarded if it was not crystal clear to avoid blood contamination. CSF was frozen immediately and stored at $-80^{\circ} \mathrm{C}$ until use. CSF samples were pooled and were mixed with $4 \mu \mathrm{l}$ of $\times 4$ sample buffer, heated at $90^{\circ} \mathrm{C}$ for 10 minutes, and separated by 4 to $12 \%$ sodium dodecyl sulfate (SDS)-polyacrylamide gel electrophoresis. They were then transferred to polyvinylidene difluoride (PVDF) membranes. The blotted membrane was heated in boiling PBS for 5 minutes to enhance the signal. ${ }^{23}$ The other steps were performed with primary (rabbit polyclonal anti-HMGB1, 1:1,000, Abcam) and secondary antibodies (horseradish peroxidase-conjugated antirabbit IgG, 1:3,000; Cell Signaling Technology, Danvers, MA) as described elsewhere. ${ }^{16}$ Briefly, nonspecific protein binding was blocked by incubating the PVDF membranes in 3\% bovine serum albumin in Tris-buffered saline-Tween 20 (TBS-T) for 1 hour. Membranes were incubated with rabbit polyclonal anti-HMGB1 overnight at $4^{\circ} \mathrm{C}$. The next day, after washing with TBS-T, the membrane was incubated with secondary antibody for 1 hour at room temperature. The protein bands were visualized by chemiluminescence (Super Signal West-Femto; Pierce Biotechnology, Rockford, IL). Images were captured by Kodak (Rochester, NY) 4000MM image station. Carestream (Rochester, NY, USA) Molecular Imaging Software was used for data analysis.

\section{Whisker Stimulation}

Whiskers on the left side were stimulated with a custom-made brush driven by a motor at a rate of $10 \mathrm{~Hz}$ for 5 minutes. These stimulation parameters were first shown to induce a robust cortical blood flow (CBF) increase in the contralateral barrel cortex by laser speckle imaging.

\section{Electrophysiological Recordings}

For extracranial recording of DC potentials, $2 \mathrm{Ag}-\mathrm{AgCl}$ pellet electrodes (Warner Instruments, Hamden, CT; E205, $1 \mathrm{~mm}$ diameter) were placed over the thinned skull. 
Electroencephalographic gel was applied to the electrode tip to obtain good electrical contact. For intracortical recordings, a cranial window ( $2 \mathrm{~mm}$ in diameter) was opened over the parietal cortex and its borders were sealed with dental cement. Two $0.5 \mathrm{M} \mathrm{NaCl}$-filled borosilicate glass microelectrodes were used for intracortical recordings. Microelectrodes were prepared by pulling glass capillaries (Harvard Apparatus, Holliston, MA; GC150F-10) with a horizontal puller (David Kopf Instruments, Tujunga, CA), and then the tips were broken into 5 to $10 \mu \mathrm{m}$ so that the impedance would be 0.5 to $1 \mathrm{MOhm}$. A chlorinated silver wire (Warner Instruments, AG15W, $0.375 \mathrm{~mm}$ diameter) was inserted into the glass microelectrodes. The tips of the microelectrodes were lowered $400 \mu \mathrm{m}$ deep into the cortex with a micromanipulator (Narishige, Tokyo, Japan; MMO220A). An $\mathrm{Ag}-\mathrm{AgCl}$-plated disk electrode was placed under the neck muscles to be used as the ground lead. All the electrodes were connected to a data acquisition system (Powerlab 8/30; ADInstruments, Bella Vista, Australia) via gold pins and jacks (Warner Instruments, WC1-10 and PJ1-10). Signals were digitized, displayed, and stored on a computer.

\section{Assessment of the CSD Threshold}

Cotton balls soaked with increasing concentrations of $\mathrm{KCl}$ $(0.05,0.075,0.1,0.15,0.25 \mathrm{M})$ were applied on intact dura over the frontal cortex 5 minutes apart. The first concentration that induced a CSD was taken as the threshold. During preliminary experiments, we observed that the $\mathrm{KCl}$-induced CSD threshold was dependent on the location of the burr hole where the $\mathrm{KCl}$-soaked cotton ball applied, and it could be lowered by dural damage and bleedings. Therefore, we discarded animals even with a minor dural damage, and used a standard location and size for the cotton ball. $\mathrm{KCl}$ was applied with a calibrated micropipette in a fixed volume $(10 \mu \mathrm{l})$. To obtain a highly reproducible threshold, we also used male mice and allowed them free access to food until the experiment to minimize potential variations that might be caused by hormonal changes and fasting.

\section{Glucose and Lactate Superfusion}

After intracortical placement of glass electrodes, $10 \mu \mathrm{l}$ of either D-glucose (0.5M; Sigma, Saint-Quentin-Fallavier, France), Lglucose (0.5 M; Sigma, Dorset, UK), L-lactate (0.5 M; Sigma, Buchs, Switzerland), or D-lactate (0.5 M; Sigma, Buchs, Switzerland) was applied for 10 minutes before recordings. The solutions were left over the dura through the entire recording. In these experiments, the CSDs were recorded by intracortical electrodes because intracortical electrodes allowed recording more focally from the cortex superfused and also eliminated the possibility of contact of the surface pellet electrodes with the superfusion fluid leaking through the cranial window despite measures taken.

\section{Antisense Knockdown of MCT2}

MCT2 antisense ODN (MCT2-ODN; 5'-GACTCTGATGGCATTTCTGAG-3') and relative scrambled ODN (SC2-ODN; 5'-GGTTTACGAGTCGTCCGTAAT-3'), which showed no homology to any mammalian sequence, were previously used in the brain in vivo with success by Suzuki et al. ${ }^{4}$ To protect ODNs from nuclease degradation, ODNs were phosphorothioated on the 3 terminal bases at each end. ODNs were purchased from Gene Link (Hawthorne, NY, USA) as reverse phase cartridge-purified. ODNs were dissolved in PBS, $\mathrm{pH} 7.4$. After anesthesia, $4 \mathrm{nmol} / \mu \mathrm{l}$ of ODN solution was injected intracortically $1 \mathrm{~mm}$ deep at 2 different points at a rate of $0.1 \mu \mathrm{l} /$ min by a 26-gauge Hamilton syringe. Coordinates of the injection sites were: $0.5 \mathrm{~mm}$ posterior, $2 \mathrm{~mm}$ lateral to bregma, and $1 \mathrm{~mm}$ anterior, $2 \mathrm{~mm}$ lateral to lambda. The needle was removed slowly over 5 minutes to allow for the diffusion of the ODNs into cortical layers. The CSD threshold was assessed with the above protocol 1 hour after injection.

\section{Sleep Deprivation}

Mice were housed in a reverse 12-hour light-dark cabinet, and they were kept in this condition for at least 1 week for adjustment. On the day of the experiment, mice were kept awake for 6 hours from the beginning of the light period using the "gentle sleep deprivation" (GSD) method described by Tobler et al. ${ }^{24}$ Briefly, animals were kept awake by providing new nesting material or new objects into their home cage when they adopted a sleeping posture of $>5$ to 7 seconds of immobility. Mice were not touched or stimulated by moving the cage to avoid stress as far as possible (hence, this method of keeping mice awake is called gentle). Control mice were left undisturbed in their home cages and sacrificed at the same time point. These mice were used in experiments to test the CSD threshold.

For detecting sleep deprivation-induced Panx transcription, 14 adult male C57BL6/j mice (8-10 weeks old, 22-25g; Charles River, Saint-Germain-Nuelles, France) were single housed in their home cage with a 12-hour light/dark cycle (lights on at 07:00, Zeitgeber time 0 [ZT0]) in standard conditions (temperature at $23 \pm 1^{\circ} \mathrm{C}$, food and water ad libitum). Following 7 days of habituation, mice were randomly divided into 2 groups ( $\mathrm{n}=7$ per group). One group underwent a 6 hour GSD beginning at ZT0 (SD6 group), and in the other group mice were left undisturbed for 6 hours (ZT6). Mice of each group were alternatively sacrificed by decapitation. These experiments were performed at the Brain Mind Institute in Lausanne, Switzerland by J.-M.P. according to the European Community's Council Directive (86/609/EEC) and with the authorization of the veterinary services of the Canton of Vaud (No. 224).

\section{Panx Gene Expression Analysis}

For each mouse, brain was rapidly dissected out on ice and cerebral cortex was homogenized (Ultraturax; IKA, Staufen, Germany) in ice-cold TRIzol and stored at $-80^{\circ} \mathrm{C}$ until RNA extraction. Total RNA was extracted from tissue samples according to the TRIzol manufacturer's protocol. The concentration of each sample was measured with NanoDrop (NanoDrop Technologies, Wilmington, DE), and the purity was assessed 
using the ratios of optic density at 260/280 $\mathrm{nm}$ and 260/ $230 \mathrm{~nm}$.

The first strand of cDNA was synthesized from 200ng of total RNA using TaqMan RT reagents (Applied Biosystems, Foster City, CA) after incubation for 45 minutes at $48^{\circ} \mathrm{C}$ followed by 5 minutes at $95^{\circ} \mathrm{C}$ and finally stored at $4{ }^{\circ} \mathrm{C}$. Then, $2 \mu \mathrm{l}$ of RT reagents were added to $0.5 \mu \mathrm{l}$ of forward and reverse primers and to $23 \mu \mathrm{l}$ of Sybr-Green PCR MasterMix (Applied Biosystems) following the manufacturer's instructions. Forty cycles of amplification were then performed in an ABI Prism 7900 (Applied Biosystems) with 384-well plate, which allowed the analysis of 3 genes simultaneously including $\beta$-actin for normalization in each plate. Each RT (from the cortex of 1 animal) was tested in triplicate. Primer sequences were designed using Primer Express (3.0) software (Applied Biosystems). Oligonucleotide primers have been synthesized by Microsynth (Balgach, Switzerland) and designed according to the published cDNA sequences (National Center for Biotechnology Information database). The forward and reverse primers for Panx-1 were $5^{\prime}$-CCCACGTCCCTACAGACCAA-3' and 5'-AGTTCT TCTCCCCATTGTTTGC-3', respectively. The forward and reverse primers for Panx-2 were 5'-CTGCTGGTCACC

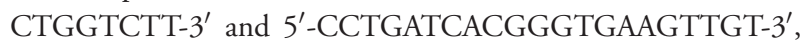
respectively. B-Actin, a housekeeping gene classically used in sleep deprivation experiments, was used as internal control to normalize data. The forward and reverse primers for $B$-actin

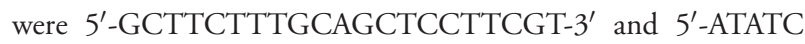
GTCATCCATGGCGAAC-3', respectively. Finally, data were obtained using SDS sequence detector software (Applied Biosystems). Expression level of target genes was normalized by $\beta$ actin level according to geNorm software (v3.3; https://genorm. cmgg.be/). Results were finally expressed as percentage of mean change ( \pm standard error of the mean $[\mathrm{SEM}]$ ) relative to the control group (ZT6).

\section{Detection of Extracellular $\mathrm{K}^{+}$by Asante Potassium Green-4 Fluoroprobe}

To detect the extracellular $\mathrm{K}^{+}$changes after $\mathrm{DAB}$ injection or GSD, a set of experiments was performed with a fluorescent potassium indicator, Asante Potassium Green (APG)-4 (TEF Labs, Austin, TX). APG-4 was prepared in 2\% DMSO and artificial CSF (aCSF) to yield a final concentration of $250 \mu \mathrm{M}$. Anesthetized (urethane) mice were placed in a stereotactic frame. A cranial window was opened over the right barrel cortex leaving dura mater intact, sealed with dental acrylic, and filled with aCSF to form a pool and closed with a coverslip. After obtaining baseline fluorescent images under a stereomicroscope (SMZ 1000; Nikon, Tokyo, Japan), the aCSF was replaced with APG-4 solution for 1 hour. At the end of the 1hour incubation period, the dye was washed out by irrigating with aCSF and then the cranial window was refilled with aCSF. Subsequently, whiskers on the left side were stimulated at $10 \mathrm{~Hz}$ for 5 minutes. Time series images were taken for 30 seconds before stimulation, 5 minutes during stimulation, and 1.5 minutes after the end of stimulation. This cycle of imaging was repeated 3 times with 5-minute intervals. Epifluorescent imaging was performed with $488 / 517 \mathrm{~nm}$ excitation and $540 \mathrm{~nm}$ emission filter sets by a monochrome DS-Qi1mc CCD camera (Nikon) set at 250-millisecond exposure time. Images were captured with NIS Elements Software (Nikon). This whisker stimulation protocol was performed on 3 groups $(n=3$ mice per group): naive mice, DAB-treated mice (incubation with solution containing $0.25 \mathrm{M} \mathrm{DAB}$ in $250 \mu \mathrm{M}$ APG-4 for 1 hour), and mice subjected to 6-hour GSD. Baseline images obtained before APG-4 application were subtracted mathematically from all time series images, and every 10 subtracted images were averaged by NIS Elements $3.2 \mathrm{AR}$ to minimize the pulsation artifacts. Two regions of interest were chosen over barrel cortex, and fluorescent intensity changes were calculated by dividing all images by basal fluorescent intensity $(\Delta \mathrm{F} / \mathrm{Fo})$. In a separate group of mice treated with topical DAB in APG-4 solution or only APG-4 solution through a cranial window over right parietal cortex, we measured the duration of APG-4 fluorescence increase during passage of a CSD wave evoked by $\mathrm{KCl}(1 \mathrm{M}$; $\mathrm{n}=3$ /group).

\section{Statistical Analyses}

Electrophysiological properties of CSD and duration of CBF changes are expressed as mean \pm SEM. Multiple group comparisons were performed by means of Kruskal-Wallis variance analysis followed by Mann-Whitney $U$ test. Threshold values are expressed as median. Analyses were performed using Fisher exact test. For Panx transcription experiments, the normalized mRNA levels of each gene in the GSD and control groups was statistically compared using a 1-way analysis of variance. Similar variances in each group were assessed using the Bartlett test. A Bonferroni multiple comparison test was used as post hoc test. Prism 5 software (GraphPad Software, La Jolla, CA) was used for calculations. A $p$ value $\leq 0.05$ was regarded as statistically significant.

\section{Results}

\section{Inhibition of Glycogen Use Opens Neuronal Panx-1 Channels}

We used a well-characterized glycogen phosphorylase inhibitor, DAB, to suppress glycogen use during synaptic activity in mice under anesthesia. We examined Panx-1 activation 1 hour after i.c.v. administration of DAB $(0.25 \mathrm{M}$ in $0.75 \mu \mathrm{l})$ slowly injected within 10 minutes with a micromanipulator through a cannula placed 1 day prior to the experiment to minimize the effect of any potential injection-induced injury. It had been established that 1 hour was required for an effective suppression of glycogen use in astrocytes by DAB. ${ }^{4,25}$ The membrane impermeable fluorescent agent PI was slowly administered i.c.v. 1 hour after DAB through the same cannula to monitor Panx-1 channel activation as large pore openings allow fluorescent dyes smaller than $1 \mathrm{KDa}$ to enter the cell as shown in vivo as well as in vitro. ${ }^{16,26,27}$ Five minutes were allowed for distribution and detectable 

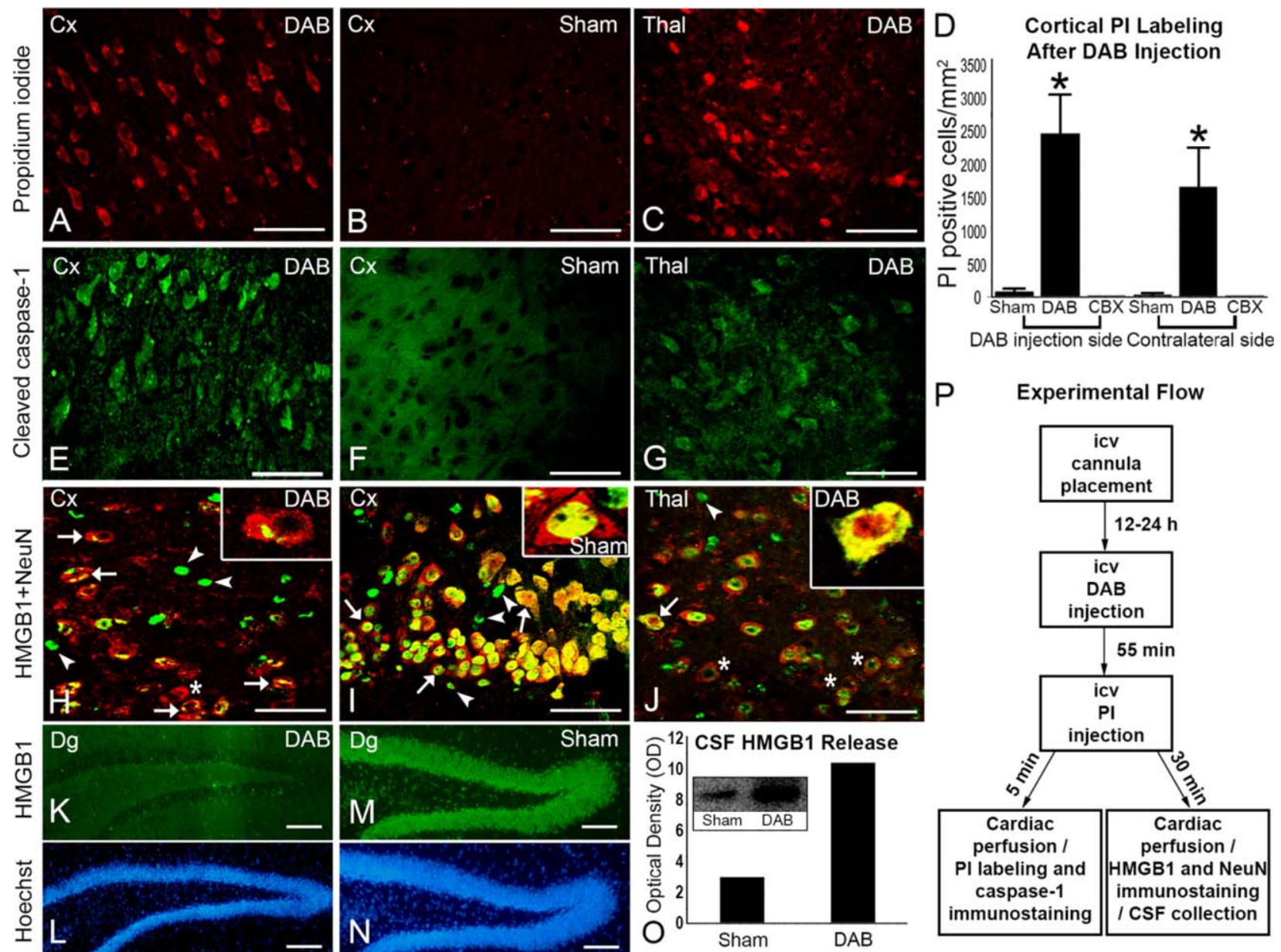

FIGURE 1: Neuronal stress induced by inhibition of glycogen utilization activates Panx1-inflammasome pathway in vivo in the mouse brain. Brains were fixed by intracardiac perfusion of paraformaldehyde 1 hour after intracerebroventricular (i.c.v.) injection of 1,4-dideoxy-1,4-imino-D-arabinitol (DAB), a glycogen phosphorylase inhibitor. First row: Membrane impermeable dye propidium iodide ( $\mathrm{Pl}$; red), which normally does not label cells (sham, B), fluxed into neurons after DAB treatment throughout the brain. (A, C) Areas from the cortex (Cx) and thalamus (Thal) are illustrated. (D) PI influx was completely inhibited by carbenoxolone (CBX), a Panx-1 channel inhibitor; ${ }^{\star} p<0.05$ compared to sham and CBX-treated groups. Second row: DAB activated the inflammasome pathway as illustrated by the appearance of the cleaved form of caspase-1 (green, E, G). Third row: HMGB1, which is normally confined to the nucleus (I, sham) of neurons identified with NeuN staining (arrows) as well as nonneuronal cells (arrowheads), was either translocated to cytoplasm (perinuclear immunostaining in $\mathrm{H}$ and $\mathrm{J}$, arrows; see insets for larger magnification) or massively released below the immunohistochemically detectable levels after DAB (asterisks in $H$ and J). HMGB1 was not released from non-neuronal cells and remained nuclear (arrowheads in $\mathrm{H}$ and J). The bottom 2 rows illustrate massive release of HMGB1 from dentate gyrus (Dg) granular neurons (visualized with nuclear Hoechst staining) after DAB (K-N; note loss of immunofluorescence from the granular neurons compared with the sham control). The coronal sections in sham and DAB-treated mice were not obtained from the same rostrocaudal level; hence, hippocampal sizes differ. (O) HMGB1 levels in the cerebrospinal fluid (CSF) also increased after DAB as detected by Western blotting. (P) The experimental flowchart; DAB and PI were injected i.c.v. through the cannula placed a day before. Scale bars $=100 \mu \mathrm{m}$. [Color figure can be viewed at www.annalsofneurology.org]

influx of PI to cells before sacrifice by intracardiac perfusion fixation, which terminates cellular activities in situ, and hence, prevents changes in the brain that might be induced by anoxia and hypoperfusion during sacrifice. The inhibition of glycogen use by DAB (but not i.c.v. saline injection) led to widespread PI influx to neurons $(\mathrm{NeuN}+$ cells $)$ in cortical and subcortical areas $(n=3$; Fig 1). The intensity of labeling paralleled DAB distribution after i.c.v. injection, being more intense on the injection side hemisphere as well as in neurons closer to the injected ventricle. In the sham group, we found PI labeling only around the cannula tract but not other brain areas in animals that were subjected to the same procedures except $D A B$ injection $(n=3)$. Further supporting the connection between $\mathrm{DAB}$ and activation of Panx-1 channels, CBX; (slowly administered i.c.v. through the cannula 10 minutes before PI) completely inhibited PI influx in another set of DAB-injected mice $(\mathrm{n}=3)$. The i.c.v. dose of CBX used had previously been shown to block Panx-1 channels without affecting gap 

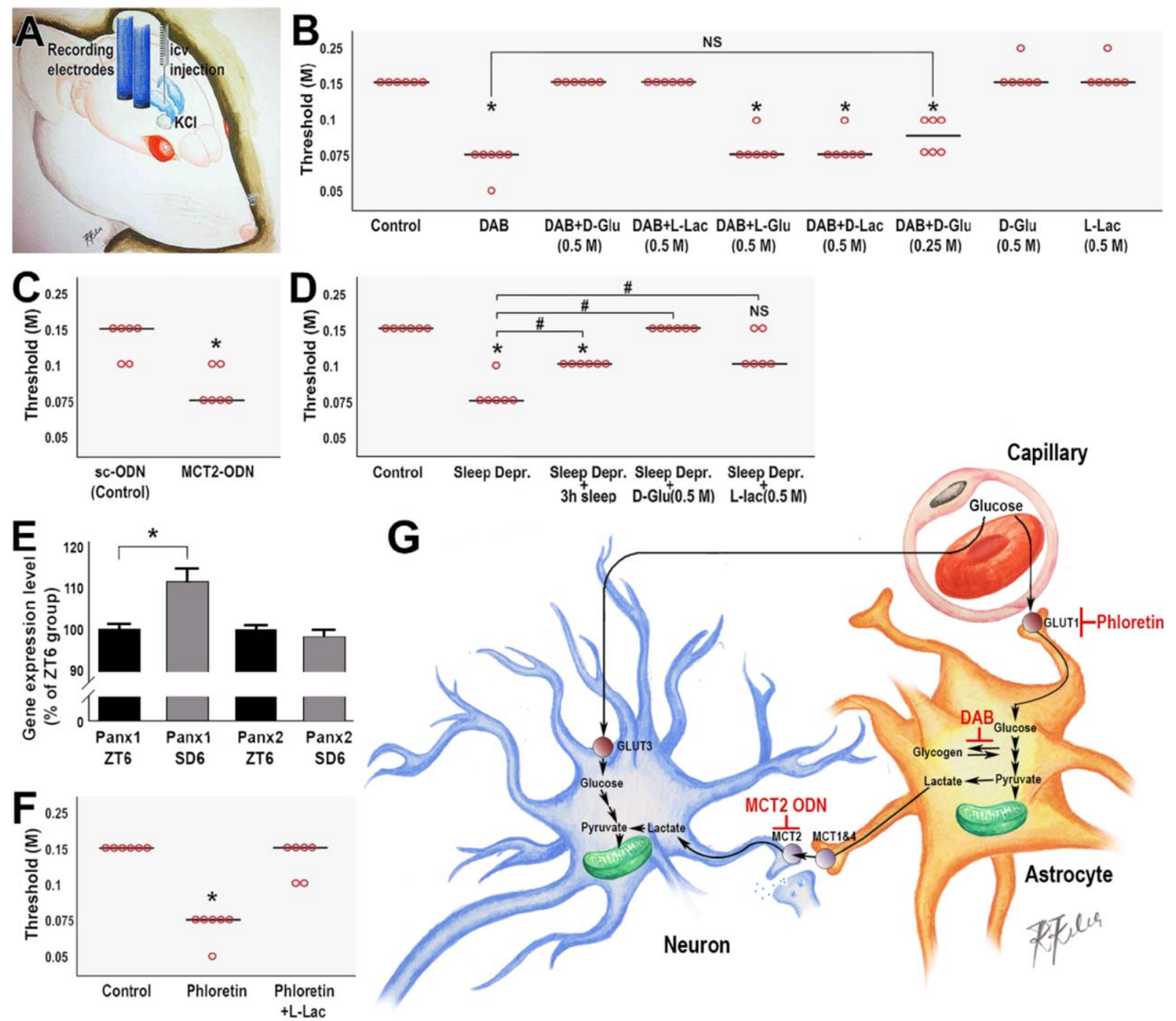

FIGURE 2: Suppression of glycogen, lactate, or glucose utilization in the cortex reduces cortical spreading depression (CSD) induction threshold. (A) $\mathrm{KCl}$-induced CSDs were recorded over the skull to avoid any nonphysiological perturbations that may affect cortex. (B) By standardizing conditions that may affect CSD generation, a highly reproducible threshold was obtained with $0.15 \mathrm{M}$ $\mathrm{KCl}$ application over the frontal dura. Inhibition of glycogen use by 1,4-dideoxy-1,4-imino-D-arabinitol (DAB) significantly (asterisks, compared to control) reduced the CSD threshold 1 hour after its intracerebroventricular (i.c.v.) injection. This effect was reversed by D-glucose (D-Glu; $0.5 \mathrm{M}$ ) or L-lactate (L-Lac; $0.5 \mathrm{M})$ superfusion over the dura but not by their optical isomers. On an equicaloric basis to $0.5 \mathrm{M}$ L-lactate, $0.25 \mathrm{M}$ D-glucose was not effective in reversing DAB's effect (*significantly reduced compared to control and not significantly [NS] different from the DAB-treated group). Without DAB treatment, L-lactate or D-glucose superfusion alone did not affect the CSD threshold. Black bars depict the median $\mathrm{KCl}$ concentration, and circles represent the individual values. (C) Supporting a role for the glycogen-derived L-lactate supply from astrocyte endfeet to synapses, knocking down the astrocyte-to-neuron lactate transporter MCT2 with antisense oligodeoxynucleotides (MCT2-ODN) but not by scrambled oligodeoxynucleotides (sc-ODN) significantly (asterisk, compared to control) reduced the CSD threshold. (D) Further supporting the importance of glycogen in preventing CSD induction, sleep deprivation for 6 hours significantly (asterisks) reduced the CSD threshold, which was significantly (hash symbols, compared to sleep deprivation group) restored by 3 hours of sleep as well as by D-glucose $(0.5 \mathrm{M})$ or L-lactate $(0.5 \mathrm{M})$ superfusion. NS = not significantly different from control. (E) Sleep deprivation induces Panx1 mRNA transcription. Bars illustrate the expression level of mRNAs encoding Panx-1 and Panx-2 in the cortex at the end of 6 hours of sleep deprivation (SD6). Compared with circadian control at Zeitgeber time 6 (ZT6; ie, 6 hours after the beginning of the light period), Panx-1 mRNA expression was significantly induced (asterisk; $p<0.0001$ ), whereas the mRNA encoding Panx-2 exhibited no significant changes. (F) Inhibition of glucose transport from the abluminal site of vessels to astrocytes by phloretin administered i.c.v. (hence, blood to endothelial glucose transport was not affected) significantly (asterisk, compared to control) reduced the CSD threshold, which was reversed by L-lactate (0.5M) superfusion. (G) Schema illustrating glucose supply to the brain, glycogen use, and the astrocyte-neuron lactate shuttle as well as the critical steps exhibited in the above experiments. [Color figure can be viewed at www.annalsofneurology.org] 
junction communication in the mouse brain in vivo. ${ }^{16,28}$ $\mathrm{DAB}$ injection also caused activation of the downstream inflammatory pathway from Panx-1 as shown by appearance of the cleaved active form of caspase- 1 and loss of nuclear HMGB1 staining in neurons with immunohistochemistry ( $\mathrm{n}=3$ for each group) as well as by release of HMGB1 to CSF as detected with Western blotting (CSF from 3 mice were pooled each for $\mathrm{DAB}$ and saline injection groups).

\section{Inhibition of Glycogen Use by DAB or Sleep Deprivation Lowers CSD Threshold}

We observed spontaneous CSDs in 3 of 8 mice within 24 to 55 minutes after $\mathrm{DAB}$ administration, possibly caused by reduced uptake of $\mathrm{K}^{+}$and glutamate to astrocytes. ${ }^{14}$ Accordingly, we investigated the effect of DAB on the CSD induction threshold in mice in which spontaneous CSDs were not observed during continuous DC potential recordings starting after $\mathrm{DAB}$ injection. Moreover, the $\mathrm{CBF}$ response recorded by laser speckle determined whether any CSDs had preceded the one evoked by $\mathrm{KCl}$, as $\mathrm{CBF}$ responses to the first and second CSDs are clearly distinguishable in the mouse. ${ }^{29}$ We used increasing concentrations of $\mathrm{KCl}$ to detect the CSD induction threshold, because we obtained a highly reproducible threshold value at $0.15 \mathrm{M} \mathrm{KCl}$ in naive control mice, unlike the highly variable threshold observed with electrical stimulation reported by several laboratories. ${ }^{30-33}$ Strictly applying the measures described in Materials and Methods, we were able to evoke the first CSD with $0.15 \mathrm{M} \mathrm{KCl}$ in every mouse in the control group $(n=15)$. After establishing a highly reproducible normal standard, we tested the effect of DAB on the CSD induction threshold and modulation of this action by glucose and lactate in groups of 6 mice in which no spontaneous CSD was observed. We found that i.c.v. injection of $\mathrm{DAB}(0.25 \mathrm{M}$ in $0.75 \mu \mathrm{l}$ saline $)$ with a high precision micromanipulator slowly over 8 to 10 minutes 1 hour prior to the test significantly lowered the CSD threshold to $0.075 \mathrm{M} \mathrm{KCl}$ compared to i.c.v. vehicleinjected sham controls (threshold $=0.15 \mathrm{M}, p=0.001$; Fig 2B). Next, we investigated whether the synaptic energy insufficiency caused by preventing glycogenolysis could be reversed by superfusing the cortex with concentrated glucose solution $(0.5 \mathrm{M})$ starting 10 minutes before recording. As glucose could penetrate through the dura, we did not remove the dura to maintain the cortical physiology. D-Glucose $(0.5 \mathrm{M})$ completely reversed the DAB-induced threshold drop, suggesting that the effect of DAB was due to lack of sufficient energy substrate to match the energy demand for clearing extracellular $\mathrm{K}^{+}$ and glutamate and preventing synchronized depolarization, leading to CSD. The effect of glucose was not due to an increased osmolarity, because superfusion with L-glucose of the same concentration failed to reverse the DAB effect. Superfusion with an equimolar concentration of L-lactate $(0.5 \mathrm{M})$ also reversed the effect of DAB, whereas its optical isomer was ineffective. On an equicaloric basis, glucose $(0.25 \mathrm{M})$ was less effective in reversing DAB's effect possibly because, unlike lactate, it is preferentially used for glycogen synthesis before being used as a fuel owing to significant differences in the reaction rates of enzymes involved in glycogen synthesizing and glycolytic pathways. ${ }^{34}$ Further supporting the important role of lactate as an energy substrate needed to prevent synchronized depolarization and CSD, knocking down MCT2 with an antisense ODN injected intracortically where recordings were performed significantly lowered the CSD induction threshold. The control scrambled antisense oligonucleotide had no significant effect $(n=6$ each for ODN and scrambled ODN groups; see Fig 2C). The ODN sequence used and injection 1 hour before the synaptic activity had been previously established to reduce expression of MCT2 by Western blotting and to impair long-term memory formation. ${ }^{4}$

Because GSD hampers glycogen use by inducing transcriptional changes in several enzymes and proteins involved in glycogen metabolism in astrocytes, ${ }^{19-21}$ we next tested whether GSD of 6 hours, a physiological disturbance, could also modify the CSD threshold. The term "gentle" here refers to gentle stimulation of the animal to keep it awake without inducing stress; hence, GSD of 6 hours duration is highly reminiscent of a common form of lack of sleep (insomnia) in humans. GSD significantly reduced the CSD induction threshold compared to the control group $(p=0.001)$. This effect was reversed by 3 hours of sleep and also by $\mathrm{D}$-glucose or Llactate superfusion, suggesting that the sleep deprivationinduced suppression of glycogen use caused the threshold change by leading to synaptic energy substrate deficiency ( $n=6$ for each group; see Fig 2D).

Because GSD induces rapid transcriptional changes in several genes involved in glycogen metabolism, which may create stress on excitatory transmission by hampering $\mathrm{K}^{+}$and glutamate clearance, we also looked at the expression level of mRNAs encoding Panx-1 (a stress sensor) and Panx-2 in the cortex at the end of 6 hours of GSD. Compared with circadian control at ZT6 (ie, 6 hours after the beginning of the light period), Panx-1 mRNA expression was significantly induced by $12 \pm 2.8 \%(p<0.0001)$, whereas the mRNA encoding Panx-2 exhibited no significant changes $(-2 \pm 1.3 \%$; see Fig 2E). 

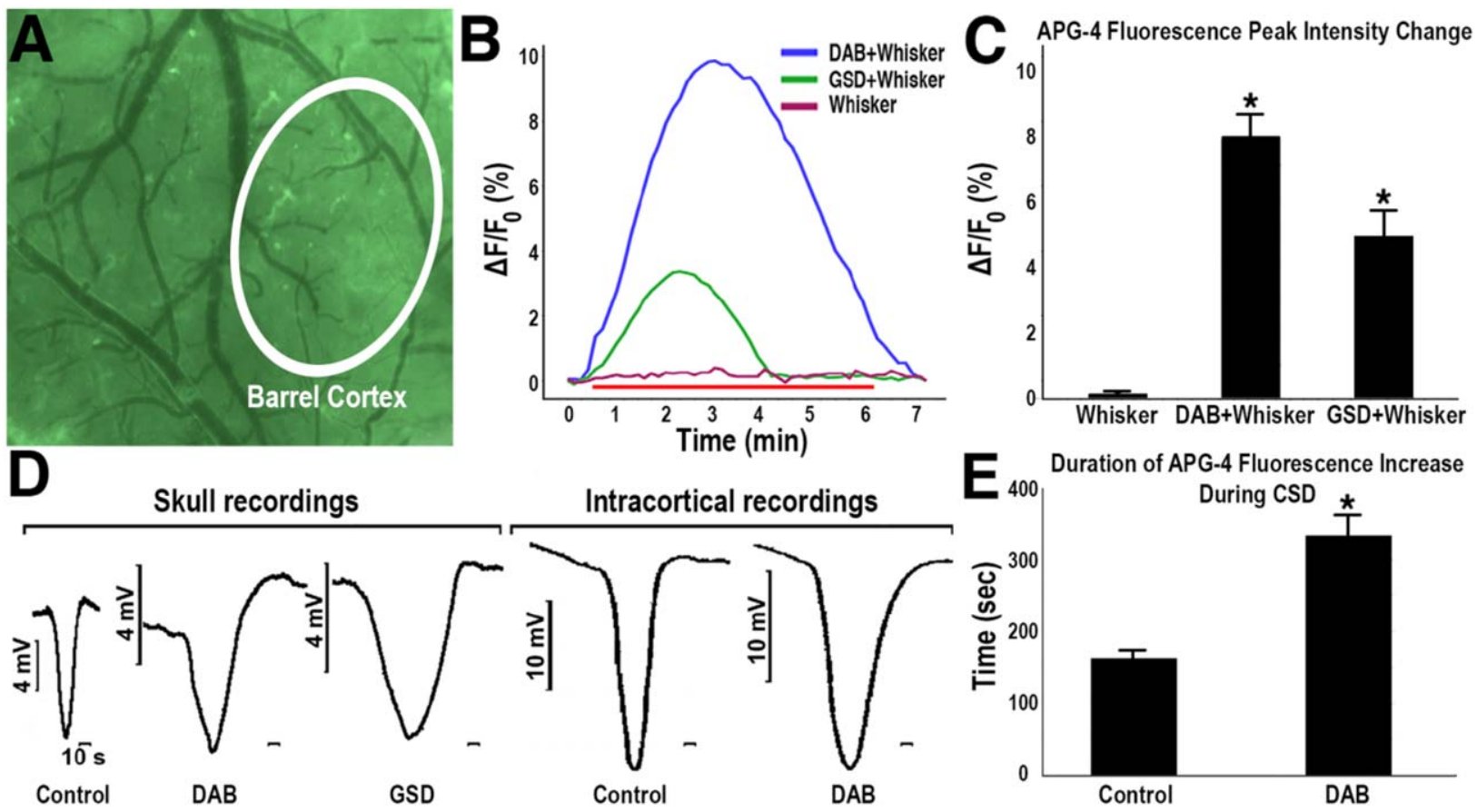

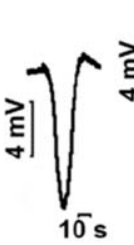

Control

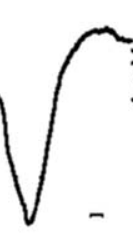

DAB

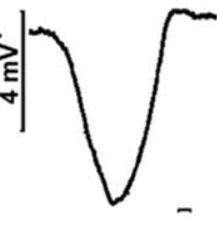

GSD

FIGURE 3: Pharmacological inhibition of glycogen utilization with 1,4-dideoxy-1,4-imino-D-arabinitol (DAB) or gentle sleep deprivation (GSD) reduces potassium uptake during sensory (whisker) stimulation. Extracellular $\mathrm{K}^{+}$was monitored by a $\mathrm{K}^{+}$-sensitive/selective fluorescent dye (Asante Potassium Green [APG]-4). (A) APG-4 fluorescence captured through cranial window from a mouse under anesthesia. APG-4 was applied over the intact dura. The white line marks the borders of the barrel cortex (Cx) where extracellular $\mathrm{K}^{+}$changes were monitored. (B) The representative APG-4 fluorescence intensity changes during whisker stimulation for 5 minutes (red horizontal bar) under normal conditions (cherry red line) or after 6 hours of sleep deprivation (green line) or 1 hour after topical DAB administration (blue line). Whereas whisker stimulation did not cause any significant extracellular $\mathrm{K}^{+}$changes owing to rapid removal of the released $\mathrm{K}^{+}$, it caused a dramatic $\mathrm{K}^{+}$accumulation after GSD or in the presence of $\mathrm{DAB}$ due to inefficient $\mathrm{K}^{+}$uptake. $\Delta \mathrm{F} / \mathrm{Fo}$ : Fluorescent intensity changes were calculated by dividing images by basal fluorescent intensity. (C) The mean \pm standard error changes in peak APG-4 fluorescence intensity during whisker stimulation in 3 groups of mice ( $\mathrm{n}=3$ per group, ${ }^{*} p<0.05$ compared to untreated controls). (D) Direct current potential recordings 1 hour after DAB injection or 6 hours of GSD revealed that the cortical spreading depression (CSD) duration was significantly prolonged, conforming with the idea that disruption of glycogen utilization slows $\mathrm{K}^{+}$uptake. (E) DAB also prolonged the duration of $\mathrm{K}^{+}$rise during CSD as detected by changes in APG-4 fluorescence intensity $\left({ }^{*} p<0.05\right)$. [Color figure can be viewed at www.annalsofneurology.org]

\section{DAB or Sleep Deprivation Reduced $K^{+}$ Clearance during Synaptic Activity}

We have adapted a recently developed $\mathrm{K}^{+}$-fluoroprobe (APG-4) to monitor extracellular $\mathrm{K}^{+}$changes during whisker stimulation in vivo through a cranial window. First, we monitored the $\mathrm{K}^{+}$rise during whisker stimulation over the barrel cortex area, which displayed little change in fluorescence $(0.15 \pm 0.04 \%$ increase $)$. However, in the presence of DAB, APG-4 fluorescence intensity changed from a hardly noticeable rise to a robust increase $(7.9 \pm 0.6 \%)$ during whisker stimulation. This confirms that $\mathrm{K}^{+}$uptake was significantly slowed during synaptic activity when glycogen mobilization was inhibited by DAB. Similarly, a striking $\mathrm{K}^{+}$rise $(4.8 \pm 1.3 \%$ increase in fluorescence intensity) was observed during 5minute whisker stimulation in sleep-deprived mice tested at the end of 6-hour GSD (Fig 3A-C). In line with these findings, both DAB and GSD prolonged the duration of CSDs without changing the CSD propagation speed and frequency (Table). We confirmed that prolongation of the CSD duration was caused by an extended $\mathrm{K}^{+}$rise during CSD by comparing APG-4 fluorescence intensity change during CSD evoked by $\mathrm{KCl}(1 \mathrm{M})$ in DABtreated and untreated control mice $(333 \pm 28$ vs $162 \pm 11$ seconds, $p=0.05$; see Fig 3D, E and Table).

\section{Inhibition of Vascular Glucose Transport Lowers CSD Threshold}

Next, we specifically tested the effect of inhibition of glucose transport from blood into astrocytes on the CSD threshold, as glucose is necessary for glycogen synthesis. Because systemic administration of insulin has several peripheral and central actions other than reducing glucose supply to brain, ${ }^{35}$ we chose to directly inhibit glucose transport to the brain from the abluminal surface of the endothelium, and selectively reduce glucose supply to astrocytes and neurons by i.c.v. administration of glucose transport protein type-1 (GLUT-1) inhibitor phloretin without significantly compromising endothelial metabolism. ${ }^{36}$ Unlike its vehicle (DMSO, $\left.1 \mu \mathrm{l}\right)$, phloretin (2M 
TABLE . Electrophysiological Characteristics of CSDs in DAB-Treated or Sleep-Deprived Mice

\begin{tabular}{llllll} 
Characteristic & $\begin{array}{l}\text { Vehicle, } \\
\text { Skull }\end{array}$ & $\begin{array}{l}\text { DAB, } \\
\text { Skull }\end{array}$ & GSD, Skull & $\begin{array}{l}\text { Vehicle, } \\
\text { Intracortical }\end{array}$ & $\begin{array}{l}\text { DAB, } \\
\text { Intracortical }\end{array}$ \\
\hline Duration of half maximum, s & $27 \pm 9$ & $45 \pm 10^{\mathrm{a}}$ & $48 \pm 3^{\mathrm{a}}$ & $22 \pm 6$ & $55 \pm 7^{\mathrm{a}}$ \\
\hline Total duration, s & $104 \pm 23$ & $156 \pm 26^{\mathrm{a}}$ & $182 \pm 19^{\mathrm{a}}$ & $81 \pm 6$ & $172 \pm 19^{\mathrm{a}}$ \\
\hline Propagation speed, $\mathrm{mm} / \mathrm{min}$ & $3.9 \pm 1.0$ & $4.2 \pm 0.7$ & $4.4 \pm 0.3$ & $4.1 \pm 0.3$ & $4.0 \pm 0.3$ \\
CSD frequency, $\mathrm{n} / \mathrm{h}$ & $7 \pm 1$ & $8 \pm 2$ & $8 \pm 2$ & $9 \pm 2$ & $9 \pm 1$
\end{tabular}

Both DAB and GSD significantly prolonged the duration of $\mathrm{KCl}$-induced CSDs compared to controls. $\mathrm{n}=6$ for each group. Values are presented as mean \pm standard error of the mean. No statistically significant differences were observed in the propagation speed and CSD frequency within 1 hour of recording.

${ }^{\mathrm{a}} p<0.05$.

$\mathrm{CSD}=$ cortical spreading depression; $\mathrm{DAB}=1,4$-dideoxy-1,4-imino-D-arabinitol; $\mathrm{GSD}=$ gentle sleep deprivation.

in $1 \mu \mathrm{l}$ ) significantly lowered the CSD threshold 30 minutes after i.c.v. injection, suggesting that a constant supply of glucose to astrocytes and neurons is needed to prevent CSD generation ( $\mathrm{n}=6$ for each group; see Fig 2F). Interestingly, contrary to their deficiency illustrated by several approaches above, excess glucose and lactate supplementation had no effect on the CSD threshold. Naive animals superfused with D-glucose or L-lactate did not show significantly different thresholds when compared to the control group $(p=0.317$ and $p=0.317$, respectively; see Fig $2 \mathrm{~B}$ ), suggesting that under resting conditions synaptic energy metabolism is balanced not to allow CSD generation ( $\mathrm{n}=6$ for each group).

\section{Discussion}

Our data show that inhibition of glycogen use in the intact brain in vivo causes Panx-1 large pore channel opening in neurons. Glycogen granules in perisynaptic astrocyte endfeet around excitatory synapses are used for uptake of glutamate and $\mathrm{K}^{+}$released during synaptic activity. ${ }^{8,37,38}$ Because glycogenolysis is stimulated by even small increases in $\left[\mathrm{K}^{+}\right]_{\mathrm{e}}$, glycogen appears to be a preferential energy substrate for maintaining low synaptic $\left[\mathrm{K}^{+}\right]_{\mathrm{e}}$ levels. Accordingly, inhibition of glycogen use by $\mathrm{DAB}$ or $\mathrm{SD}$ causes a rise of $\left[\mathrm{K}^{+}\right]_{\mathrm{e}}$ toward higher values than normal during synaptic activity, as clearly shown with our in vivo studies using a $\mathrm{K}^{+}$-sensitive/selective fluoroprobe. Studies in brain slices have established that accumulation of $\left[\mathrm{K}^{+}\right]_{\mathrm{e}}>10 \mathrm{mM}$ can activate Panx-1 large pore channel. ${ }^{11}$ Owing to very narrow extracellular space around synapses, inefficient $\mathrm{K}^{+}$removal caused by DAB may readily result in $\mathrm{K}^{+}$accumulation to concentrations $>10 \mathrm{mM}$ and activate Panx-1 channels. Similarly to $\mathrm{K}^{+}$, extracellular glutamate buildup may contribute to activation of Panx- 1 channels by activating N-methyl-D- aspartate (NMDA) receptors. ${ }^{39,40}$ In addition to the demonstration of Panx-1 activation during synaptic energy insufficiency in vivo, these findings also provide strong support to the idea that glycogen-derived energy is indispensable for maintaining low concentrations of synaptic extracellular $\mathrm{K}^{+}$and glutamate throughout the brain. ${ }^{8,37,41}$ Therefore, we conclude that when glycogen availability decreases, as for example due to prolonged and/or intense neural stimulation or to sleep deprivation, neuronal Panx-1 channels may be activated under physiological conditions, as also suggested by induction of Panx-1 expression by sleep deprivation. Cleavage of caspase-1 in these neurons and release of HMGB1 suggest that Panx-1 activation may serve to report the stress created by synaptic metabolism-activity mismatch.

Data from familial hemiplegic migraine (FHM) 1 and 2 knockin mice indicate that unregulated glutamate increases in the synaptic cleft of recurrent synapses between pyramidal cells may culminate in simultaneous depolarization of an aggregate of neurons and trigger CSD. ${ }^{42,43}$ Excess extracellular glutamate may also trigger regenerative glutamate release by stimulating presynaptic NMDA receptors, which may contribute to rapid escalation of synchronized network excitation to a spreading depolarization wave. ${ }^{44}$ Knockin mice carrying human PQ type calcium channel mutations (FHM1) have a lower CSD threshold compared to their wild-type littermates ${ }^{45}$ possibly because of excess glutamate release during synaptic activity. In FHM2, haploinsufficiency of ATP1A2 (the gene that encodes a form of the $\mathrm{Na}^{+} / \mathrm{K}^{+}$ ATPase-alpha 2 selectively localized in astrocytes and shown to be activated by glutamate uptake into astrocytes) ${ }^{46}$ also leads to a reduced capacity to remove glutamate and to a lesser extent $\mathrm{K}^{+}$; hence, FHM2 knockin mice exhibit a low CSD threshold. ${ }^{30}$ Therefore, one may 
posit that conditions that decrease glycogen utilization may similarly predispose the cortical neurons to generate CSD during intense/prolonged synaptic activity due to accumulation of glutamate and $\mathrm{K}^{+}$. Our findings strongly support this view by showing that interfering with glycogen use either with pharmacological blockade (DAB) or with sleep deprivation lowers the CSD induction threshold. Prolongation of the duration of CSDs and associated extracellular $\mathrm{K}^{+}$rise ${ }^{14,47-50}$ supports this idea. The predisposition to CSD generation created by $\mathrm{DAB}$ or sleep deprivation is likely to be caused by insufficient energy supply during synaptic activity, because the CSD threshold drop was completely reversed by supplying extra lactate or glucose to the interstitium. Similarly, the lower CSD threshold observed after inhibition of glucose transfer to astrocytes with phloretin or knocking down MCT2, the transporter that supplies lactate to preand postsynaptic compartments, is in line with the idea that an insufficient energy supply to match synaptic activity leads to uncontrolled synchronous excitability. ${ }^{51,52}$ Decrease in the CSD threshold induced by knocking down MCT2 suggests that sufficient energy supply to pre- and postsynaptic compartments is important to prevent CSD generation in addition to maintaining low extracellular glutamate and $\mathrm{K}^{+}$concentrations. Restoration of the reduced CSD threshold induced by insufficient glycogen availability with lactate as well as glucose conforms with the view that glycogen is used to match the rapidly emerging energy demand during intensive synaptic activity by supplying lactate as well as glucose to pre- and postsynaptic compartments. ${ }^{53}$ It appears so that the finetuning of the excitatory synapses terminating on excitatory as well as inhibitory neurons within a network to prevent synchronized network depolarization (which may spread to the neighboring networks once generated) is energy dependent such that insufficient acceleration of energy substrate supply from perisynaptic astrocyte processes may lead to buildup of glutamate and $\mathrm{K}^{+}$concentrations to supranormal levels in the synaptic cleft, favoring synchronized excitation in cortical networks, as observed in FHM knockin animals.

Altogether, these observations with DAB, sleep deprivation, MCT2 knockdown, and phloretin conform with the hypothesis that glycogen in astrocyte processes is strategically positioned to secure the energy demand required for rapid removal of glutamate and $\mathrm{K}^{+}$from synaptic cleft (see Fig 2G). ${ }^{54}$ Glucose transported from the circulation can then be directly metabolized by astrocytes and used to restore the glycogen stores. ${ }^{55}$ This metabolic safety system over the excitatory synaptic activity may ensure that the cortical networks sustain their activity in a desynchronized state. Given the importance of
CSD in the development of migraine aura and headache, therefore, an adequate glycogen turnover appears to be critical such that an insufficient mobilization of glycogen created by for example sleep deprivation or conditions that reduce glucose transport to the brain, such as GLUT-1 deficiency syndrome, ${ }^{56}$ may predispose to CSD generation and headache (migraine with aura). ${ }^{16}$ Interestingly, when $\mathrm{K}^{+}$and glutamate accumulation caused by insufficient glycogen availability induces Panx-1 activation without CSD, then the inflammatory cascade downstream from Panx-1 activation may trigger headache without aura. Supporting this view, several cases of GLUT-1 deficiency syndrome have been reported to suffer from migraine with and without aura attacks, which were successfully prevented with ketogenic diet. ${ }^{56,57} \mathrm{Sim}-$ ilarly, patients with monocarboxylate transporter 1 (which transports lactate from astrocyte to synapses) deficiency have been reported to have migraines. ${ }^{58}$ Therefore, our results may have important clinical implications. The genetic findings from familial monogenic disorders can be extended to nonfamilial migraine cases by including several other genes involved in synaptic energy metabolism. Several biological factors on a genetically susceptible background may account for why insufficient sleep triggers migraine to varying degrees among patients. Ketogenic diet could be tested on sleep-deprived patients to see whether it prevents migraine attacks by providing ketone bodies as alternative energy substrates for synaptic transmission. Similarly, the effect of glucose supplement or ketogenic diet on reducing the photosensitivity in migraine patients could also be tested.

In conclusion, our findings demonstrate how Panx1 channels can be activated and upregulated under stressful metabolic conditions during synaptic activity and suggest that migraine with and without aura could be triggered by synaptic metabolic stress.

\section{Acknowledgment}

This work was supported by the Turkish Academy of Sciences (T.D.), Hacettepe University Research Fund (013 D07 105 001-258, T.D.), Swiss National Science Foundation (3100AO-108336/1, P.J.M.) and a grant from the Préfargier Foundation (P.J.M).

We thank M. Yilmaz for his expert help with Western blotting, Ş. Hanalioğlu for his advice on APG-4 experiments, J. Gyger for his excellent technical expertise in real-time polymerase chain reaction experiments, and $\mathrm{D}$. A. Madencioglu and B. Uzay for their help with sleep deprivation experiments. 


\section{Author Contributions}

T.D., H.K., K.K., P.J.M., J.-M.P., and Y.G.-O. contributed to the study concept and design; K.K., H.K., B.D.D., E.E.-K., J.-M.P., and A.C. performed data acquisition and analysis; T.D., K.K., A.C., J.-M.P., and H.K. contributed to drafting the manuscript and preparing the figures; T.D. conceived the hypothesis.

\section{Potential Conflicts of Interest}

Nothing to report.

\section{References}

1. Magistretti PJ. Neuron-glia metabolic coupling and plasticity. Exp Physiol 2011;96:407-410.

2. Belanger M, Allaman I, Magistretti PJ. Brain energy metabolism: focus on astrocyte-neuron metabolic cooperation. Cell Metab $2011 ; 14: 724-738$

3. Obel LF, Müller MS, Walls AB, et al. Brain glycogen-new perspectives on its metabolic function and regulation at the subcellular level. Front Neuroenergetics 2012;4:3.

4. Suzuki A, Stern SA, Bozdagi O, et al. Astrocyte-neuron lactate transport is required for long-term memory formation. Cell 2011; 144:810-823.

5. Schousboe A, Sickmann HM, Walls $A B$, et al. Functional importance of the astrocytic glycogen-shunt and glycolysis for maintenance of an intact intra/extracellular glutamate gradient. Neurotox Res 2010;18:94-99.

6. Schousboe A, Sickmann HM, Bak LK, et al. Neuron-glia interactions in glutamatergic neurotransmission: roles of oxidative and glycolytic adenosine triphosphate as energy source. J Neurosci Res 2011;89:1926-1934.

7. Dinuzzo M, Mangia S, Maraviglia B, Giove F. The role of astrocytic glycogen in supporting the energetics of neuronal activity. Neurochem Res 2012;37:2432-2438.

8. Sickmann HM, Walls $A B$, Schousboe $A$, et al. Functional significance of brain glycogen in sustaining glutamatergic neurotransmission. J Neurochem 2009;109(suppl 1):80-86.

9. Silverman WR, de Rivero Vaccari JP, Locovei S, et al. The pannexin 1 channel activates the inflammasome in neurons and astrocytes. J Biol Chem 2009:284:18143-18151.

10. Wang J, Ambrosi C, Qiu F, et al. The membrane protein Pannexin 1 forms two open-channel conformations depending on the mode of activation. Sci Signal 2014;7:ra69.

11. Jackson DG, Wang J, Keane RW, et al. ATP and potassium ions: a deadly combination for astrocytes. Sci Rep 2014;4:4576.

12. MacVicar BA, Thompson RJ. Non-junction functions of pannexin-1 channels. Trends Neurosci 2010;33:93-102.

13. Sandilos JK, Bayliss DA. Physiological mechanisms for the modulation of pannexin 1 channel activity. J Physiol 2012;590:6257-6266.

14. Pietrobon D, Moskowitz MA. Chaos and commotion in the wake of cortical spreading depression and spreading depolarizations. Nat Rev Neurosci 2014:15:379-393.

15. Ayata C, Lauritzen M. Spreading depression, spreading depolarizations, and the cerebral vasculature. Physiol Rev 2015;95:953993.

16. Karatas H, Erdener SE, Gursoy-Ozdemir Y, et al. Spreading depression triggers headache by activating neuronal Panx 1 channels. Science. 2013;339:1092-1095.
17. Chen SP, Qin T, Seidel JL, et al. Inhibition of the P2X7-PANX1 complex suppresses spreading depolarization and neuroinflammation. Brain 2017;140:1643-1656.

18. Lateef $T$, Swanson S, Cui L, et al. Headaches and sleep problems among adults in the United States: findings from the National Comorbidity Survey-Replication study. Cephalalgia 2011;31:648653.

19. Petit JM, Tobler I, Allaman I, et al. Sleep deprivation modulates brain mRNAs encoding genes of glycogen metabolism. Eur $J$ Neurosci 2002;16:1163-1167.

20. Petit JM, Tobler I, Kopp C, et al. Metabolic response of the cerebral cortex following gentle sleep deprivation and modafinil administration. Sleep 2010;33:901-908.

21. Morgenthaler FD, Lanz BR, Petit JM, et al. Alteration of brain glycogen turnover in the conscious rat after $5 \mathrm{~h}$ of prolonged wakefulness. Neurochem Int 2009;55:45-51.

22. Magistretti PJ, Allaman I. A cellular perspective on brain energy metabolism and functional imaging. Neuron 2015;86:883-901.

23. Ida N, Hartmann T, Pantel J, et al. Analysis of heterogeneous A4 peptides in human cerebrospinal fluid and blood by a newly developed sensitive Western blot assay. J Biol Chem 1996;271: 22908-22914.

24. Tobler I, Deboer T, Fischer M. Sleep and sleep regulation in normal and prion protein-deficient mice. J Neurosci 1997;17:18691879

25. Walls $A B$, Sickmann $H M$, Brown $A$, et al. Characterization of 1,4 dideoxy-1,4-imino-d-arabinitol (DAB) as an inhibitor of brain glycogen shunt activity. J Neurochem 2008;105:1462-1470.

26. Pelegrin $P$, Surprenant $A$. Pannexin-1 mediates large pore formation and interleukin-1beta release by the ATP-gated P2X7 receptor. EMBO J 2006;25:5071-5082

27. Dahl G, Qiu F, Wang J. The bizarre pharmacology of the ATP release channel pannexin1. Neuropharmacology 2013;75:583593.

28. Wang J, Jackson DG, Dahl G. The food dye FD\&C Blue No. 1 is a selective inhibitor of the ATP release channel Panx1. J Gen Physiol 2013:141:649-656.

29. Ayata C, Shin HK, Salomone S, et al. Pronounced hypoperfusion during spreading depression in mouse cortex. J Cereb Blood Flow Metab 2004;24:1172-1182.

30. Leo L, Gherardini L, Barone V, et al. Increased susceptibility to cortical spreading depression in the mouse model of familial hemiplegic migraine type 2. PLoS Genet 2011;7:e1002129.

31. Hoffmann U, Sukhotinsky I, Eikermann-Haerter K, Ayata C. Glucose modulation of spreading depression susceptibility. J Cereb Blood Flow Metab 2013;33:191-195.

32. Ayata C. Pearls and pitfalls in experimental models of spreading depression. Cephalalgia 2013;33:604-613.

33. Brennan KC, Romero Reyes M, Lopez Valdes HE, et al. Reduced threshold for cortical spreading depression in female mice. Ann Neurol 2007;61:603-606.

34. Shulman RG, Rothman DL. Homeostasis and the glycogen shunt explains aerobic ethanol production in yeast. Proc Natl Acad Sci USA 2015;112:10902-10907.

35. Cardoso S, Correia S, Santos RX, et al. Insulin is a two-edged knife on the brain. J Alzheimers Dis 2009:18:483-507.

36. Gibbs ME, Hutchinson DS, Summers RJ. Role of betaadrenoceptors in memory consolidation: beta3-adrenoceptors act on glucose uptake and beta2-adrenoceptors on glycogenolysis. Neuropsychopharmacology 2008;33:2384-2397.

37. Xu J, Song $D, X u e ~ Z$, et al. Requirement of glycogenolysis for uptake of increased extracellular $\mathrm{K}+$ in astrocytes: potential 
implications for $\mathrm{K}+$ homeostasis and glycogen usage in brain Neurochem Res 2013;38:472-485.

38. Maxwell DS, Kruger L. The fine structure of astrocytes in the cerebral cortex and their response to focal injury produced by heavy ionizing particles. J Cell Biol 1965;25:141-157.

39. Weilinger NL, Tang PL, Thompson RJ. Anoxia-induced NMDA receptor activation opens pannexin channels via Src family kinases. J Neurosci 2012;32:12579-12588.

40. Thompson RJ, Jackson MF, Olah ME, et al. Activation of pannexin-1 hemichannels augments aberrant bursting in the hippocampus. Science 2008;322:1555-1559.

41. Dienel GA, Cruz NF. Contributions of glycogen to astrocytic energetics during brain activation. Metab Brain Dis 2015;30:281-298.

42. Vecchia D, Pietrobon D. Migraine: a disorder of brain excitatoryinhibitory balance? Trends Neurosci 2012;35:507-520.

43. Moskowitz MA, Bolay H, Dalkara T. Deciphering migraine mechanisms: clues from familial hemiplegic migraine genotypes. Ann Neurol 2004;55:276-280.

44. Zhou N, Rungta RL, Malik A, et al. Regenerative glutamate release by presynaptic NMDA receptors contributes to spreading depression. J Cereb Blood Flow Metab 2013;33:1582-1594.

45. van den Maagdenberg AM, Pietrobon D, Pizzorusso T, et al. A Cacna1a knockin migraine mouse model with increased susceptibility to cortical spreading depression. Neuron 2004;41:701710.

46. Pellerin L, Magistretti PJ. Glutamate uptake stimulates $\mathrm{Na}+, \mathrm{K}+$ ATPase activity in astrocytes via activation of a distinct subunit highly sensitive to ouabain. J Neurochem 1997;69:2132-2137.

47. Lauritzen M, Dreier JP, Fabricius $M$, et al. Clinical relevance of cortical spreading depression in neurological disorders: migraine, malignant stroke, subarachnoid and intracranial hemorrhage, and traumatic brain injury. J Cereb Blood Flow Metab 2011;31:17-35.
48. Fordsmann JC, Ko RW, Choi HB, et al. Increased 20-HETE synthesis explains reduced cerebral blood flow but not impaired neurovascular coupling after cortical spreading depression in rat cerebral cortex. J Neurosci 2013;33:2562-2570.

49. Chang JC, Brennan KC, He D, et al. A mathematical model of the metabolic and perfusion effects on cortical spreading depression. PLoS One 2013;8:e70469.

50. Brinley FJ Jr, Kandel ER, Marshall WH. Potassium outflux from rabbit cortex during spreading depression. J Neurophysiol 1960; 23:246-256.

51. Stobart JL, Anderson CM. Multifunctional role of astrocytes as gatekeepers of neuronal energy supply. Front Cell Neurosci 2013; $7: 38$

52. Nagase M, Takahashi $Y$, Watabe AM, et al. On-site energy supply at synapses through monocarboxylate transporters maintains excitatory synaptic transmission. J Neurosci 2014;34:2605-2617.

53. Pellerin L, Magistretti PJ. Sweet sixteen for ANLS. J Cereb Blood Flow Metab 2012;32:1152-1166.

54. Richter K, Hamprecht B, Scheich H. Ultrastructural localization of glycogen phosphorylase predominantly in astrocytes of the gerbil brain. Glia 1996;17:263-273.

55. Barros LF, Bittner CX, Loaiza A, Porras $\mathrm{OH}$. A quantitative overview of glucose dynamics in the gliovascular unit. Glia 2007;55: 1222-1237.

56. De Giorgis V, Veggiotti P. GLUT1 deficiency syndrome 2013: current state of the art. Seizure 2013;22:803-811.

57. Mohammad SS, Coman D, Calvert S. Glucose transporter 1 deficiency syndrome and hemiplegic migraines as a dominant presenting clinical feature. J Paediatr Child Health 2014;50:1025-1026.

58. van Hasselt PM, Ferdinandusse S, Monroe GR, et al. Monocarboxylate transporter 1 deficiency and ketone utilization. N Engl J Med 2014;371:1900-1907 